\title{
Bounded-Degree Graphs have Arbitrarily Large Geometric Thickness
}

\author{
János Barát* \\ Bolyai Institute \\ University of Szeged \\ Szeged, Hungary \\ barat@math.u-szeged.hu \\ Jiří Matoušek \\ Department of Applied Mathematics and \\ Institute for Theoretical Computer Science \\ Charles University \\ Prague, Czech Republic \\ matousek@kam.mff.cuni.cz \\ David R. Wood ${ }^{\dagger}$ \\ Departament de Matemática Aplicada II \\ Universitat Politècnica de Catalunya \\ Barcelona, Spain \\ david.wood@upc.edu
}

Submitted: Sep 9, 2005; Accepted: Dec 22, 2005; Published: Jan 7, 2006

Mathematics Subject Classification: 05C62, 05C10

\begin{abstract}
The geometric thickness of a graph $G$ is the minimum integer $k$ such that there is a straight line drawing of $G$ with its edge set partitioned into $k$ plane subgraphs. Eppstein [Separating thickness from geometric thickness. In Towards a Theory of Geometric Graphs, vol. 342 of Contemp. Math., AMS, 2004] asked whether every graph of bounded maximum degree has bounded geometric thickness. We answer this question in the negative, by proving that there exists $\Delta$-regular graphs with
\end{abstract}

*Research of János Barát was supported by a Marie Curie Fellowship of the European Community under contract number HPMF-CT-2002-01868 and by the OTKA Grant T.49398.

${ }^{\dagger}$ Research of David Wood is supported by the Government of Spain grant MEC SB2003-0270, and by the projects MCYT-FEDER BFM2003-00368 and Gen. Cat 2001SGR00224. 
arbitrarily large geometric thickness. In particular, for all $\Delta \geq 9$ and for all large $n$, there exists a $\Delta$-regular graph with geometric thickness at least $c \sqrt{\Delta} n^{1 / 2-4 / \Delta-\epsilon}$. Analogous results concerning graph drawings with few edge slopes are also presented, thus solving open problems by Dujmović et al. [Really straight graph drawings. In Proc. 12th International Symp. on Graph Drawing (GD '04), vol. 3383 of Lecture Notes in Comput. Sci., Springer, 2004] and Ambrus et al. [The slope parameter of graphs. Tech. Rep. MAT-2005-07, Department of Mathematics, Technical University of Denmark, 2005].

\section{Introduction}

A drawing of an (undirected, finite, simple) graph represents each vertex by a distinct point in the plane, and represents each edge by a simple closed curve between its endpoints, such that the only vertices an edge intersects are its own endpoints. Two edges cross if they intersect at a point other than a common endpoint. A drawing is plane if no two edges cross.

The thickness of an (abstract) graph $G$ is the minimum number of planar subgraphs of $G$ whose union is $G$. Thickness is a classical and widely studied graph parameter; see the survey [23]. The thickness of a graph drawing $D$ is the minimum number of plane subgraphs of $D$ whose union is $D$. Every planar graph can be drawn with its vertices at prespecified locations $[14,25]$. It follows that a graph with thickness $k$ has a drawing with thickness $k$ [14]. However, in such a representation the edges might be highly curved ${ }^{1}$.

This motivates the notion of geometric thickness, which is a central topic of this paper. A drawing is geometric, also called a geometric graph, if every edge is represented by a straight line segment. The geometric thickness of a graph $G$ is the minimum thickness of a geometric drawing of $G$; see $[7,10,11,13,15]$. Geometric thickness was introduced by Kainen [18] under the name real linear thickness.

Consider the relationship between the various thickness parameters and maximum degree. A graph with maximum degree at most $\Delta$ is called degree- $\Delta$. Wessel [35] and Halton [14] independently proved that the thickness of a degree- $\Delta$ graph is at most $\left\lceil\frac{\Delta}{2}\right\rceil$, and Sýkora et al. [31] proved that this bound is tight. Duncan et al. [11] proved that the geometric thickness of a degree-4 graph is at most 2. Eppstein [13] asked whether graphs of bounded degree have bounded geometric thickness. The first contribution of this paper is to answer this question in the negative.

Theorem 1. For all $\Delta \geq 9$ and $\epsilon>0$, for all large $n>n(\epsilon)$ and $n \geq c \Delta$, there exists a $\Delta$-regular $n$-vertex graph with geometric thickness at least

$$
c \sqrt{\Delta} n^{1 / 2-4 / \Delta-\epsilon}
$$

for some absolute constant $c$.

\footnotetext{
${ }^{1}$ In fact, a polyline drawing of a random perfect matching on $n$ vertices in convex position almost certainly has $\Omega(n)$ bends on some edge [25].
} 
A number of notes on Theorem 1 are in order. Eppstein [13] proved that geometric thickness is not bounded by thickness. In particular, there exists a graph with thickness 3 and arbitrarily large geometric thickness. Theorem 1 and the above result of Wessel [35] and Halton [14] imply a similar result (with a shorter proof). Namely, there exists a 9-regular graph with thickness at most 5 and with arbitrarily large geometric thickness.

A book embedding is a geometric drawing with the vertices in convex position. The book thickness of a graph $G$ is the minimum thickness of a book embedding of $G$. Book thickness is also called page-number and stack-number; see [9] for over fifty references on this topic. By definition, the geometric thickness of $G$ is at most the book thickness of $G$. On the other hand Eppstein [12] proved that there exists a graph with geometric thickness 2 and arbitrarily large book thickness; also see [5, 6]. Thus book thickness is not bounded by any function of geometric thickness.

Theorem 1 is analogous to a result of Malitz [19], who proved that there exists $\Delta$ regular $n$-vertex graphs with book thickness at least $c \sqrt{\Delta} n^{1 / 2-1 / \Delta}$. Malitz's proof is based on a probabilistic construction of a graph with certain expansion properties. The proof of Theorem 1 is easily adapted to prove Malitz's result for $\Delta \geq 3$. The difference in the bounds $\left(n^{1 / 2-4 / \Delta}\right.$ and $\left.n^{1 / 2-1 / \Delta}\right)$ is caused by the difference between the number of order types of point sets in general and convex position ( $\approx n^{4 n}$ and $\left.n !\right)$. Malitz [19] also proved an upper bound of $\mathcal{O}(\sqrt{m}) \subseteq \mathcal{O}(\sqrt{\Delta n})$ on the book thickness, and thus the geometric thickness, of $m$-edge graphs.

The other contributions of this paper concern geometric graph drawings with few slopes. Wade and Chu [33] defined the slope-number of a graph $G$ to be the minimum number of distinct edge slopes in a geometric drawing of $G$. If $G$ has a vertex of degree $d$, then the slope-number of $G$ is at least $\lceil d / 2\rceil$. Dujmovic et al. [8] asked whether every graph with bounded maximum degree has bounded slope-number. Since edges with the same slope do not cross, the geometric thickness of $G$ is at most the slope-number of $G$. Thus Theorem 1 immediately implies a negative answer to this question for $\Delta \geq 9$, which is improved as follows ${ }^{2}$.

Theorem 2. For all $\Delta \geq 5$ there exists a $\Delta$-regular graph with arbitrarily large slopenumber.

The proofs of Theorems 1 and 2 are simple. We basically show that there are more graphs with bounded degree than with bounded geometric thickness (or slope-number). Our counting arguments are based on two tools from the literature that are introduced in Section 2. Theorem 1 is then proved in Section 3. In Section 4 we study a graph parameter recently introduced by Ambrus et al. [3] that is similar to the slope-number, and we solve two of their open problems. The proofs will also serve as a useful introduction to the proof of Theorem 2, which is presented in Section 5.

\footnotetext{
${ }^{2}$ Note that Pach and Pálvölgyi [24] independently obtained the following strengthening of Theorem 2: For all $\Delta \geq 5$ there exists a $\Delta$-regular $n$-vertex graph whose slope-number is at least $n^{1 / 2-1 /(\Delta-2)-o(1)}$. The proof is also based on Lemma 1 .
} 


\section{Tools}

All of our results are based on the following lemma, versions of which are due to Petrovskil and Oleı̆nik [26], Milnor [22], Thom [32] and Warren [34]. The precise version stated here is by Pollack and Roy [27]; see [20]. Let $\mathcal{P}=\left(P_{1}, P_{2}, \ldots, P_{t}\right)$ be a system of $d$-variate real polynomials. A vector $\sigma \in\{-1,0,+1\}^{t}$ is a sign pattern of $\mathcal{P}$ if there exists an $x \in \mathbb{R}^{d}$ such that the sign of $P_{i}(x)$ is $\sigma_{i}$, for all $i=1,2, \ldots, t$.

Lemma 1 ([27]). Let $\mathcal{P}=\left(P_{1}, P_{2}, \ldots, P_{t}\right)$ be a system of $d$-variate real polynomials, each of degree at most $D$. Then the number of sign patterns of $\mathcal{P}$ is at most

$$
\left(\frac{50 D t}{d}\right)^{d}
$$

Some of our proofs only need sign patterns that distinguish between zero and nonzero values. In this setting, Rónyai et al. [29] gave a better bound with a short proof; see [20].

Our second tool is a corollary of more precise bounds due to Bender and Canfield [4], Wormald [36], and McKay [21]; see Appendix A.

Lemma 2 ([4, 21, 36]). For all integers $\Delta \geq 1$ and $n \geq c \Delta$, the number of labelled $\Delta$-regular $n$-vertex graphs is at least

$$
\left(\frac{n}{3 \Delta}\right)^{\Delta n / 2}
$$

for some absolute constant $c$.

\section{Geometric Thickness: Proof of Theorem 1}

Observe that a geometric drawing with thickness $k$ can be perturbed so that the vertices are in general position (that is, no three vertices are collinear). Thus in this section we consider point sets in general position without loss of generality. (We cannot make this assumption for drawings with few slopes.)

Lemma 3. The number of labelled n-vertex graphs with geometric thickness at most $k$ is at most $472^{k n} n^{4 n+o(n)}$.

Proof. Let $P$ be a fixed set of $n$ labelled points in general position in the plane. Ajtai et al. [1] proved that there are at most $c^{n}$ plane geometric graphs with vertex set $P$, where $c \leq 10^{13}$. Santos and Seidel [30] proved that we can take $c=472$. A geometric graph with vertex set $P$ and thickness at most $k$ consists of a $k$-tuple of plane geometric graphs with vertex set $P$. Thus $P$ admits at most $472^{k n}$ geometric graphs with thickness at most $k$.

Let $P=\left(p_{1}, p_{2}, \ldots, p_{n}\right)$ and $Q=\left(q_{1}, q_{2}, \ldots, q_{n}\right)$ be two sets of $n$ points in general position in the plane. Then $P$ and $Q$ have the same order type if for all indices $i<j<k$ we turn in the same direction (left or right) when going from $p_{i}$ to $p_{k}$ via $p_{j}$ and when 
going from $q_{i}$ to $q_{k}$ via $q_{j}$. Say $P$ and $Q$ have the same order type. Then for all $i, j, k, \ell$, the segments $p_{i} p_{j}$ and $p_{k} p_{\ell}$ cross if and only if $q_{i} q_{j}$ and $q_{k} q_{\ell}$ cross. Thus $P$ and $Q$ admit the same set of (at most $472^{k n}$ ) labelled geometric graphs with thickness at most $k$ (when considering $p_{i}$ and $q_{i}$ to be labelled $i$ ). Alon [2] proved (using Lemma 1) that there are at most $n^{4 n+o(n)}$ sets of $n$ points with distinct order types. The result follows.

It is easily seen that Lemmas 2 and 3 imply a lower bound of $c(\Delta-8) \log n$ on the geometric thickness of some $\Delta$-regular $n$-vertex graph. To improve this logarithmic bound to polynomial, we now give a more precise analysis of the number of graphs with bounded geometric thickness that also accounts for the number of edges in the graph.

Lemma 4. Let $P$ be a set of $n$ labelled points in general position in the plane. Let $g(P, m)$ be the number of $m$-edge plane geometric graphs with vertex set $P$. Then

$$
g(P, m) \leq \begin{cases}\left(\begin{array}{c}
n \\
2 m
\end{array}\right) \cdot 472^{2 m} & , \text { if } m \leq \frac{n}{2} \\
472^{n} & , \text { if } m>\frac{n}{2}\end{cases}
$$

Proof. As in Lemma $3, g(P, m) \leq 472^{n}$ regardless of $m$. Suppose that $m \leq \frac{n}{2}$. An $m$-edge graph has at most $2 m$ vertices of nonzero degree. Thus every $m$-edge plane geometric graph with vertex set $P$ is obtained by first choosing a $2 m$-element subset $P^{\prime} \subseteq P$, and then choosing a plane geometric graph on $P^{\prime}$. The result follows.

Lemma 5. Let $P$ be a set of $n$ labelled points in general position in the plane. For every integer $t$ such that $\frac{2 m}{n} \leq t \leq m$, let $g(P, m, t)$ be the number of $m$-edge geometric graphs with vertex set $P$ and thickness at most $t$. Then

$$
g(P, m, t) \leq\left(\frac{c t n}{m}\right)^{2 m}
$$

for some absolute constant c.

Proof. Fix nonnegative integers $m_{1} \leq m_{2} \leq \cdots \leq m_{t}$ such that $\sum_{i} m_{i}=m$. Let $g\left(P ; m_{1}, m_{2}, \ldots, m_{t}\right)$ be the number of geometric graphs with vertex set $P$ and thickness $t$, such that there are $m_{i}$ edges in the $i$-th subgraph. Then

$$
g\left(P ; m_{1}, m_{2}, \ldots, m_{t}\right) \leq \prod_{i=1}^{t} g\left(P, m_{i}\right)
$$

Now $m_{1} \leq \frac{n}{2}$, as otherwise $m>\frac{t n}{2} \geq m$. Let $j$ be the maximum index such that $m_{j} \leq \frac{n}{2}$. By Lemma 4,

$$
g\left(P ; m_{1}, m_{2}, \ldots, m_{t}\right) \leq\left(\prod_{i=1}^{j}\left(\begin{array}{c}
n \\
2 m_{i}
\end{array}\right) 472^{2 m_{i}}\right)\left(472^{n}\right)^{t-j}
$$


Now $\sum_{i=1}^{j} m_{i} \leq m-\frac{1}{2}(t-j) n$. Thus

$$
g\left(P ; m_{1}, m_{2}, \ldots, m_{t}\right) \leq\left(\prod_{i=1}^{j}\left(\begin{array}{c}
n \\
2 m_{i}
\end{array}\right)\right)\left(472^{2 m-(t-j) n}\right)\left(472^{(t-j) n}\right) \leq 472^{2 m} \prod_{i=1}^{t}\left(\begin{array}{c}
n \\
2 m_{i}
\end{array}\right) .
$$

We can suppose that $t$ divides $2 m$. It follows (see Appendix B) that

$$
g\left(P ; m_{1}, m_{2}, \ldots, m_{t}\right) \leq 472^{2 m}\left(\begin{array}{c}
n \\
2 m / t
\end{array}\right)^{t} .
$$

It is well known [17, Proposition 1.3] that $\left(\begin{array}{l}n \\ k\end{array}\right)<\left(\frac{\mathrm{e} n}{k}\right)^{k}$, where e is the base of the natural logarithm. Thus with $k=2 m / t$ we have

$$
g\left(P ; m_{1}, m_{2}, \ldots, m_{t}\right)<\left(\frac{236 \mathrm{et} n}{m}\right)^{2 m} .
$$

Clearly

$$
g(P, m, t) \leq \sum_{m_{1}, \ldots, m_{t}} g\left(P ; m_{1}, m_{2}, \ldots, m_{t}\right)
$$

where the sum is taken over all nonnegative integers $m_{1} \leq m_{2} \leq \cdots \leq m_{t}$ such that $\sum_{i} m_{i}=m$. The number of such partitions [17, Proposition 1.4] is at most

$$
\left(\begin{array}{c}
t+m-1 \\
m
\end{array}\right)<\left(\begin{array}{c}
2 m \\
m
\end{array}\right)<2^{2 m}
$$

Hence

$$
g(P, m, t) \leq 2^{2 m}\left(\frac{236 \mathrm{et} n}{m}\right)^{2 m} \leq\left(\frac{c t n}{m}\right)^{2 m}
$$

As in Lemma 3, we have the following corollary of Lemma 5.

Corollary 1. For all integers $t$ such that $\frac{2 m}{n} \leq t \leq m$, the number of labelled $n$-vertex $m$-edge graphs with geometric thickness at most $t$ is at most

$$
n^{4 n+o(n)}\left(\frac{c t n}{m}\right)^{2 m}
$$

for some absolute constant $c$.

Proof of Theorem 1. Let $t$ be the minimum integer such that every $\Delta$-regular $n$-vertex graph has geometric thickness at most $t$. Thus the number of $\Delta$-regular $n$-vertex graphs is at most the number of labelled graphs with $\frac{1}{2} \Delta n$ edges and geometric thickness at most t. By Lemma 2 and Corollary 1,

$$
\left(\frac{n}{3 \Delta}\right)^{\Delta n / 2} \leq n^{4 n+o(n)}\left(\frac{c t}{\Delta}\right)^{\Delta n} \leq n^{4 n+\epsilon n}\left(\frac{c t}{\Delta}\right)^{\Delta n},
$$

for large $n>n(\epsilon)$. Hence $t \geq \sqrt{\Delta} n^{1 / 2-4 / \Delta-\epsilon} /(c \sqrt{3})$. 
It remains open whether geometric thickness is bounded by a constant for graphs with $\Delta \leq 8$. The above method is easily modified to prove Malitz's lower bound on book thickness that is discussed in Section 1.

Theorem 3 ([19]). For all $\Delta \geq 3$ and $n \geq c \Delta$, there exists a $\Delta$-regular n-vertex graph with book thickness at least

$$
c \sqrt{\Delta} n^{1 / 2-1 / \Delta},
$$

for some absolute constant $c$.

Proof. Obviously the number of order types for point sets in convex position is $n$ !. As in the proof of Theorem 1 ,

$$
\left(\frac{n}{3 \Delta}\right)^{\Delta n / 2} \leq n !\left(\frac{c t}{\Delta}\right)^{\Delta n} \leq n^{n}\left(\frac{c t}{\Delta}\right)^{\Delta n}
$$

Hence $t \geq \sqrt{\Delta} n^{1 / 2-1 / \Delta} /(c \sqrt{3})$. (The constant $c$ can be considerably improved here; for example, we can replace 472 by 16 .)

\section{The Slope Parameter of Ambrus et al. [3]}

Ambrus et al. [3] introduced the following slope parameter of graphs. Let $P \subseteq \mathbb{R}^{2}$ be a set of points in the plane. Let $S \subset \mathbb{R} \cup\{\infty\}$ be a set of slopes. Let $G(P, S)$ be the graph with vertex set $P$ where two points $v, w \in P$ are adjacent if and only if the slope of the line $\overline{v w}$ is in $S$. The slope parameter of a graph $G$, denoted by $\operatorname{sl}(G)$, is the minimum integer $k$ such that $G \cong G(P, S)$ for some point set $P$ and slope set $S$ with $|S|=k$. Note that sl is well-defined, since $\mathrm{sl}(G) \leq|E(G)|$. Slope parameter and slope number are not necessarily related. For example, Jamison [16] proved that the slope-number of $K_{n}$ is $n$, but the slope parameter of $K_{n}$ is 1 (just use $n$ collinear points). In this section we address the following two questions of Ambrus et al. [3]:

- what is the maximum slope parameter of an $n$-vertex graph?

- do graphs of bounded maximum degree have bounded slope parameter?

Lemma 6. The number of labelled n-vertex graphs $G$ with slope parameter $\mathrm{sl}(G) \leq k$ is at most

$$
\left(\frac{50 n^{2} k}{2 n+k}\right)^{2 n+k}
$$

Proof. Let $\mathcal{G}_{n, k}$ denote the family of labelled $n$-vertex graphs $G$ with slope parameter $\mathrm{sl}(G) \leq k$. Consider $V(G)=\{1,2, \ldots, n\}$ for every $G \in \mathcal{G}_{n, k}$. For every $G \in \mathcal{G}_{n, k}$, there is a point set $P=\left\{\left(x_{i}(G), y_{i}(G)\right): 1 \leq i \leq n\right\}$ and slope set $S=\left\{s_{\ell}(G): 1 \leq \ell \leq k\right\}$, such that $G \cong G(P, S)$, where vertex $i$ is represented by the point $\left(x_{i}(G), y_{i}(G)\right)$. Fix one such representation of $G$. Without loss of generality, $x_{i}(G) \neq x_{j}(G)$ for distinct $i$ and $j$. Thus 
every $s_{\ell}(G)<\infty$. For all $i, j, \ell$ with $1 \leq i<j \leq n$ and $1 \leq \ell \leq k$, and for every graph $G \in G_{n, k}$, we define the number

$$
P_{i, j, \ell}(G):=\left(y_{j}(G)-y_{i}(G)\right)-s_{\ell}(G) \cdot\left(x_{j}(G)-x_{i}(G)\right) .
$$

Consider

$$
\mathcal{P}:=\left\{P_{i, j, \ell}: 1 \leq i<j \leq n, 1 \leq \ell \leq k\right\}
$$

to be a set of $\left(\begin{array}{l}n \\ 2\end{array}\right) k$ degree-2 polynomials on the set of variables

$$
\left\{x_{1}, x_{2}, \ldots, x_{n}, y_{1}, y_{2}, \ldots, y_{n}, s_{1}, s_{2}, \ldots, s_{k}\right\} .
$$

Observe that $P_{i, j, \ell}(G)=0$ if and only if $i j$ is an edge of $G$ and $i j$ has slope $s_{\ell}$ in the representation of $G$.

Consider two distinct graphs $G, H \in \mathcal{G}_{n, k}$. Without loss of generality, there is an edge $i j$ of $G$ that is not an edge of $H$. Thus $\left(y_{j}(G)-y_{i}(G)\right)-s_{\ell}(G) \cdot\left(x_{j}(G)-x_{i}(G)\right)=0$ for some $\ell$, and $\left(y_{j}(H)-y_{i}(H)\right)-s_{\ell}(H) \cdot\left(x_{j}(H)-x_{i}(H)\right) \neq 0$ for all $\ell$. Hence $P_{i, j, \ell}(G)=0 \neq$ $P_{i, j, \ell}(H)$. That is, any two distinct graphs in $\mathcal{G}_{n, k}$ are distinguished by the sign of some polynomial in $\mathcal{P}$. Hence $\left|\mathcal{G}_{n, k}\right|$ is at most the number of sign patterns determined by $\mathcal{P}$. By Lemma 1 with $D=2, d=2 n+k$, and $t=\left(\begin{array}{l}n \\ 2\end{array}\right) k$ we have

$$
\left|\mathcal{G}_{n, k}\right| \leq\left(\frac{50 \cdot 2 \cdot\left(\begin{array}{c}
n \\
2
\end{array}\right) k}{2 n+k}\right)^{2 n+k}<\left(\frac{50 n^{2} k}{2 n+k}\right)^{2 n+k}
$$

In response to the first question of Ambrus et al. [3], we now prove that there exist graphs with surprisingly large slope parameter. In this paper all logarithms are binary unless stated otherwise.

Theorem 4. For all $\epsilon>0$ and for all sufficiently large $n>n(\epsilon)$, there exists an $n$-vertex graph $G$ with slope parameter

$$
\operatorname{sl}(G) \geq \frac{n^{2}}{(4+\epsilon) \log n} .
$$

Proof. Suppose that every $n$-vertex graph $G$ has slope parameter $\operatorname{sl}(G) \leq k$. There are $2^{\left(\begin{array}{c}n \\ 2\end{array}\right)}$ labelled $n$-vertex graphs. By Lemma 6,

$$
2^{\left(\begin{array}{c}
n \\
2
\end{array}\right)} \leq\left(\frac{50 n^{2} k}{2 n+k}\right)^{2 n+k}
$$

For large $n>n(\epsilon)$,

$$
2^{\left(\begin{array}{l}
n \\
2
\end{array}\right)}=\left(50 n^{2}\right)^{\left(\begin{array}{c}
n \\
2
\end{array}\right) / \log \left(50 n^{2}\right)}>\left(50 n^{2}\right)^{\left(\begin{array}{c}
n \\
2
\end{array}\right) /(2+\epsilon / 2) \log n}>\left(50 n^{2}\right)^{2 n+n^{2} /(4+\epsilon) \log n} .
$$


We have $50 n^{2} k \leq 50 n^{2}(2 n+k)$. Thus

$$
\left(50 n^{2}\right)^{2 n+n^{2} /(4+\epsilon) \log n}<2^{\left(\begin{array}{c}
n \\
2
\end{array}\right)} \leq\left(\frac{50 n^{2} k}{2 n+k}\right)^{2 n+k}<\left(50 n^{2}\right)^{2 n+k} .
$$

Hence

$$
k>\frac{n^{2}}{(4+\epsilon) \log n}
$$

The result follows.

Now we prove that the slope parameter of degree- $\Delta$ graphs is unbounded for $\Delta \geq 5$, thus answering the second question of Ambrus et al. [3] in the negative. It remains open whether $\operatorname{sl}(G)$ is bounded for degree-3 or degree-4 graphs $G$.

Theorem 5. For all $\Delta \in\{5,6,7,8\}$, for all $\epsilon$ with $0<\epsilon<\Delta-4$, and for all sufficiently large $n>n(\Delta, \epsilon)$, there exists a $\Delta$-regular $n$-vertex graph $G$ with

$$
\mathrm{sl}(G)>n^{(\Delta-4-\epsilon) / 4} .
$$

Proof. Let $k:=n^{(\Delta-4-\epsilon) / 4}$. Suppose that for some $\Delta \in\{5,6,7,8\}$, every $\Delta$-regular $n$-vertex graph $G$ has $\mathrm{sl}(G) \leq k$. By Lemmas 2 and 6 ,

$$
\left(\frac{n}{3 \Delta}\right)^{\Delta n / 2} \leq\left(\frac{50 n^{2} k}{2 n+k}\right)^{2 n+k}<(25 n k)^{2 n+k}<(25 n)^{(\Delta-\epsilon)(2 n+k) / 4}
$$

For $n>\left(3 \Delta(25)^{1-\epsilon / 2}\right)^{2 / \epsilon}$,

$$
(25 n)^{(2 \Delta-\epsilon) n / 4}<\left(\frac{n}{3 \Delta}\right)^{\Delta n / 2}<(25 n)^{(\Delta-\epsilon)(2 n+k) / 4} .
$$

Thus $2 \Delta n-\epsilon n<2 \Delta n+\Delta k-2 \epsilon n-\epsilon k$. That is, $(\Delta-\epsilon) n^{(\Delta-8-\epsilon) / 4}>\epsilon$. Thus $\Delta-8-\epsilon \geq 0$ for large $n>n(\Delta, \epsilon)$, which is the desired contradiction for $\Delta \leq 8$.

For $\Delta \geq 9$ there are graphs with linear slope parameter.

Theorem 6. For all $\Delta \geq 9$ and $\epsilon>0$, and for all sufficiently large $n>n(\Delta, \epsilon)$, there exists a $\Delta$-regular $n$-vertex graph $G$ with slope parameter

$$
\mathrm{sl}(G)>\frac{1}{4}((1-\epsilon) \Delta-8) n
$$

Proof. Suppose that every $\Delta$-regular $n$-vertex graph $G$ has $\mathrm{sl}(G) \leq \alpha n$ for some $\alpha>0$. By Lemmas 2 and 6,

$$
\left(\frac{n}{3 \Delta}\right)^{\Delta n / 2} \leq\left(\frac{50 \alpha n^{2}}{2+\alpha}\right)^{(2+\alpha) n}
$$

For $n>\left(3 \Delta \cdot 8^{1-\epsilon}\right)^{1 / \epsilon}$

$$
(8 n)^{(1-\epsilon) \Delta n / 2}<\left(\frac{n}{3 \Delta}\right)^{\Delta n / 2} \leq\left(\frac{50 \alpha n^{2}}{2+\alpha}\right)^{(2+\alpha) n}<(8 n)^{2(2+\alpha) n} .
$$


Thus

$$
\alpha>\frac{(1-\epsilon) \Delta-8}{4} .
$$

Thus $\mathrm{sl}(G) \geq \frac{1}{4}((1-\epsilon) \Delta-8) n$ for some $\Delta$-regular $n$-vertex graph $G$.

Note that the lower bound in Theorem 6 is within a factor of $2+\epsilon$ of the trivial upper bound $\mathrm{sl}(G) \leq \frac{1}{2} \Delta n$.

\section{Slope-Number: Proof of Theorem 2}

In this section we extend the method developed in Section 4 to prove a lower bound on the slope-number of graphs with bounded degree.

Lemma 7. The number of labelled $n$-vertex $m$-edge graphs with slope-number at most $k$ is at most

$$
\left(\frac{50 n^{2}(k+1)}{2 n+k}\right)^{2 n+k}\left(\begin{array}{c}
k(n-1) \\
m
\end{array}\right) .
$$

Proof. Consider $V(G)=\{1,2, \ldots, n\}$ for every labelled $n$-vertex $m$-edge graph $G$ with slope-number at most $k$. For every such graph $G$, fix a $k$-slope drawing of $G$ represented by a point set $\left\{\left(x_{i}(G), y_{i}(G)\right): 1 \leq i \leq n\right\}$ and slope set $\left\{s_{\ell}(G): 1 \leq \ell \leq k\right\}$. Thus for every edge $i j$ of $G$, the slope of the line through $\left(x_{i}(G), y_{i}(G)\right)$ and $\left(x_{j}(G), y_{j}(G)\right)$ equals $s_{\ell}(G)$ for some $\ell$. Without loss of generality, every $s_{\ell}(G)<\infty$. Define $\mathcal{P}$ as in the proof of Lemma 6. In addition, for all $i, j$ with $1 \leq i<j \leq n$, define $Q_{i, j}(G):=x_{i}(G)-x_{j}(G)$. Let $\mathcal{Q}:=\left\{Q_{i, j}: 1 \leq i<j \leq n\right\}$. By Lemma 1 with $D=2, d=2 n+k$, and $t=\left(\begin{array}{l}n \\ 2\end{array}\right)(k+1)$, the number of sign patterns of $\mathcal{P} \cup \mathcal{Q}$ is at most

$$
\left(\frac{50 n^{2}(k+1)}{2 n+k}\right)^{2 n+k}
$$

Fix a sign pattern $\sigma$ of $\mathcal{P} \cup \mathcal{Q}$. As in Lemma 6 , from $\sigma$ restricted to $\mathcal{P}$ we can reconstruct the collinear subsets of vertices. Moreover, from $\sigma$ restricted to $\mathcal{Q}$, we can reconstruct the order of the vertices within each collinear subset. Observe that at most $n-1$ edges have the same slope in a geometric drawing. Thus every $k$-slope graph representable by $\sigma$ is a subgraph of a fixed graph with at most $k(n-1)$ edges. Hence $\sigma$ corresponds to at most $\left(\begin{array}{c}k(n-1) \\ m\end{array}\right)$ labelled $k$-slope graphs on $m$ edges. The result follows.

Proof of Theorem 2. Suppose that for some $\Delta \geq 5$ and for some integer $k$, every $\Delta$-regular graph has slope-number at most $k$. By Lemmas 2 and 7 , for all $n$,

$$
\left(\frac{n}{3 \Delta}\right)^{\Delta n / 2} \leq\left(\frac{50 n^{2}(k+1)}{2 n+k}\right)^{2 n+k}\left(\begin{array}{c}
k(n-1) \\
\frac{1}{2} \Delta n
\end{array}\right) .
$$

Let $\epsilon=\epsilon(\Delta)>0$ be specified later. For large $n>n(k, \Delta, \epsilon)$ there is a constant $c=c(k, \Delta)$ such that

$$
\left(\begin{array}{c}
k(n-1) \\
\frac{1}{2} \Delta n
\end{array}\right) \leq c^{n}=n^{n / \log _{c} n}<n^{\epsilon n} .
$$


Thus

$$
\left(\frac{n}{3 \Delta}\right)^{\Delta n / 2} \leq\left(\frac{50 n^{2}(k+1)}{2 n+k}\right)^{2 n+k} n^{\epsilon n}<(25 n(k+1))^{(2+\epsilon) n+k} .
$$

For $n>\left(3 \Delta(25(k+1))^{1-\epsilon}\right)^{1 / \epsilon}$ we have

$$
(25 n(k+1))^{(1-\epsilon) \Delta n / 2}<\left(\frac{n}{3 \Delta}\right)^{\Delta n / 2}<(25 n(k+1))^{(2+\epsilon) n+k} .
$$

Thus $(1-\epsilon) \Delta n<(4+\epsilon) n+2 k$. Choose $\epsilon>0$ such that $(1-\epsilon) \Delta>4+\epsilon$. We obtain a contradiction for large $n>\frac{2 k}{(1-\epsilon) \Delta-(4+\epsilon)}$. Thus there exists a $\Delta$-regular graph with slope-number greater than $k$

It remains open whether slope-number is bounded by a constant for all degree-3 or degree-4 graphs.

\section{Acknowledgement}

Thanks to Emo Welzl and the Theory of Combinatorial Algorithms group at ETH Zürich, and to Carsten Thomassen and the Technical University of Denmark for their generous hospitality which enabled this collaboration. Thanks to Vida Dujmović, János Pach and Nick Wormald for fruitful discussions.

\section{References}

[1] Miklós Ajtai, Vašek Chvátal, Monroe M. Newborn, and Endre SzeMERÉDI. Crossing-free subgraphs. In Theory and practice of combinatorics, vol. 60 of North-Holland Math. Stud., pp. 9-12. North-Holland, 1982.

[2] Noga Alon. The number of polytopes, configurations and real matroids. Mathematika, 33(1):62-71, 1986.

[3] Gergely Ambrus, János Barát, and Péter Hajnal. The slope parameter of graphs. Tech. Rep. MAT-2005-07, Department of Mathematics, Technical University of Denmark, Lyngby, Denmark, 2005.

[4] Edward A. Bender and E. Rodney Canfield. The asymptotic number of labeled graphs with given degree sequences. J. Combin. Theory Ser. A, 24:296-307, 1978.

[5] Robin Blankenship. Book Embeddings of Graphs. Ph.D. thesis, Department of Mathematics, Louisiana State University, U.S.A., 2003.

[6] Robin Blankenship and Bogdan Oporowski. Drawing subdivisions of complete and complete bipartite graphs on books. Tech. Rep. 1999-4, Department of Mathematics, Louisiana State University, U.S.A., 1999.

[7] Michael B. Dillencourt, David Eppstein, and Daniel S. Hirschberg. Geometric thickness of complete graphs. J. Graph Algorithms Appl., 4(3):5-17, 2000. 
[8] Vida Dujmović, Matthew Suderman, and David R. Wood. Really straight graph drawings. In JÁnos PACH, ed., Proc. 12th International Symp. on Graph Drawing (GD '04), vol. 3383 of Lecture Notes in Comput. Sci., pp. 122-132. Springer, 2004.

[9] Vida Dujmović and David R. Wood. On linear layouts of graphs. Discrete Math. Theor. Comput. Sci., 6(2):339-358, 2004.

[10] Vida Dujmović and David R. Wood. Graph treewidth and geometric thickness parameters. In PATRICK HeAly, ed., Proc. 13th International Symp. on Graph Drawing (GD '05), Lecture Notes in Comput. Sci., Springer, to appear. http://arxiv.org/math/0503553.

[11] Christian A. Duncan, David Eppstein, and Stephen G. Kobourov. The geometric thickness of low degree graphs. In Proc. 20th ACM Symp. on Computational Geometry (SoCG '04), pp. 340-346. ACM Press, 2004.

[12] David Eppstein. Separating geometric thickness from book thickness, 2001. http://arXiv.org/math/0109195.

[13] David Eppstein. Separating thickness from geometric thickness. In JÁnOs PACH, ed., Towards a Theory of Geometric Graphs, vol. 342 of Contemporary Mathematics, pp. 75-86. Amer. Math. Soc., 2004.

[14] John H. Halton. On the thickness of graphs of given degree. Inform. Sci., 54(3):219-238, 1991.

[15] Joan P. Hutchinson, Thomas C. Shermer, and Andrew Vince. On representations of some thickness-two graphs. Comput. Geom., 13(3):161-171, 1999.

[16] Robert E. Jamison. Few slopes without collinearity. Discrete Math., 60:199-206, 1986.

[17] Stasys Jukna. Extremal combinatorics: with applications in computer science. Texts in Theoretical Computer Science. Springer, 2001. ISBN 3-540-66313-4.

[18] Paul C. Kainen. Thickness and coarseness of graphs. Abh. Math. Sem. Univ. Hamburg, 39:88-95, 1973.

[19] Seth M. MaLitz. Graphs with $E$ edges have pagenumber $O(\sqrt{E})$. J. Algorithms, 17(1):71-84, 1994.

[20] JiŘí MatoušEk. Lectures on Discrete Geometry, vol. 212 of Graduate Texts in Mathematics. Springer, 2002. ISBN 0-387-95373-6.

[21] Brendan D. McKay. Asymptotics for symmetric 0-1 matrices with prescribed row sums. Ars Combin., 19(A):15-25, 1985.

[22] John Milnor. On the Betti numbers of real varieties. Proc. Amer. Math. Soc., 15:275-280, 1964.

[23] Petra Mutzel, Thomas Odenthal, and Mark Scharbrodt. The thickness of graphs: a survey. Graphs Combin., 14(1):59-73, 1998. 
[24] JÁnos PACH And Dömötör PÁlvölgyi. Bounded-degree graphs can have arbitrarily large slope numbers. Electron. J. Combin., 13:N1, 2006.

[25] János Pach and Rephael Wenger. Embedding planar graphs at fixed vertex locations. Graphs Combin., 17(4):717-728, 2001.

[26] Ivan G. Petrovskĭ and Olga A. Ole Ĭnik. On the topology of real algebraic surfaces. Izvestiya Akad. Nauk SSSR. Ser. Mat., 13:389-402, 1949.

[27] Richard Pollack and Marie-Françoise Roy. On the number of cells defined by a set of polynomials. C. R. Acad. Sci. Paris Sér. I Math., 316(6):573-577, 1993.

[28] Herbert Robbins. A remark on Stirling's formula. Amer. Math. Monthly, 62:26$29,1955$.

[29] Lajos Rónyai, László Babai, and Murali K. Ganapathy. On the number of zero-patterns of a sequence of polynomials. J. Amer. Math. Soc., 14(3):717-735, 2001.

[30] Francisco Santos and Raimund Seidel. A better upper bound on the number of triangulations of a planar point set. J. Combin. Theory Ser. A, 102(1):186-193, 2003.

[31] Ondrej SÝkora, László A. SzÉkely, and Imrich VRŤo. A note on Halton's conjecture. Inform. Sci., 164(1-4):61-64, 2004.

[32] René Thom. Sur l'homologie des variétés algébriques réelles. In S. S. CAIRns, ed., Differential and Combinatorial Topology, pp. 255-265. Princeton Univ. Press, 1965.

[33] Greg A. Wade and Jiang-Hsing Chu. Drawability of complete graphs using a minimal slope set. The Computer Journal, 37(2):139-142, 1994.

[34] Hugh E. Warren. Lower bounds for approximation by nonlinear manifolds. Trans. Amer. Math. Soc., 133:167-178, 1968.

[35] Walter Wessel. Über die Abhängigkeit der Dicke eines Graphen von seinen Knotenpunktvalenzen. In 2nd Colloquium for Geometry and Combinatorics, pp. 235-238. Tech. Hochschule Karl-Marx-Stadt, 1983.

[36] Nicholas Wormald. Some problems in the enumeration of labelled graphs. Ph.D. thesis, Newcastle-upon-Tyne, United Kingdom, 1978. 


\section{A Derivation of Lemma 2}

Let $f(n, \Delta)$ denote the number of labelled $\Delta$-regular $n$-vertex graphs. The first asymptotic bounds on $f(n, \Delta)$ were independently determined by Bender and Canfield [4] and Wormald [36]. Refining these results, McKay [21] proved that for all $\Delta$ with $1 \leq \Delta<\frac{2}{9} n$,

$$
f(n, \Delta)=\frac{(\Delta n) !}{(\Delta n / 2) ! 2^{\Delta n / 2}(\Delta !)^{n} \mathrm{e}^{\left(\Delta^{2}-1\right) / 4-\mathcal{O}\left(\Delta^{3} / n\right)}} .
$$

The version of Stirling's formula due to Robbins [28] states that for all $t \geq 1$,

$$
t !=\sqrt{2 \pi t}\left(\frac{t}{\mathrm{e}}\right)^{t} \mathrm{e}^{r(t)},
$$

where $1 /(12 t+1)<r(t)<1 / 12 t$. Thus, for some constant $c$,

$$
\begin{aligned}
f(n, \Delta) & \geq \frac{\sqrt{2 \pi \Delta n}\left(\frac{\Delta n}{\mathrm{e}}\right)^{\Delta n} \mathrm{e}^{r(\Delta n)}}{\sqrt{\pi \Delta n}\left(\frac{\Delta n}{2 \mathrm{e}}\right)^{\Delta n / 2} \mathrm{e}^{r(\Delta n / 2)} 2^{\Delta n / 2} \Delta^{\Delta n} \mathrm{e}^{c \Delta^{2}}} \\
& \geq \sqrt{2}\left(\frac{n}{\Delta}\right)^{\Delta n / 2} / \exp \left(\frac{\Delta n}{2}-\frac{1}{12 \Delta n}+\frac{1}{6 \Delta n+1}+c \Delta^{2}\right) .
\end{aligned}
$$

With $n>200 c \Delta$, we have

$$
\frac{\Delta n}{2}-\frac{1}{12 \Delta n}+\frac{1}{6 \Delta n+1}+c \Delta^{2}<\frac{102 \Delta n}{200} .
$$

Thus

$$
f(n, \Delta)>\left(\frac{n}{\mathrm{e}^{1.02} \Delta}\right)^{\Delta n / 2}>\left(\frac{n}{3 \Delta}\right)^{\Delta n / 2}
$$

Lemma 2 follows.

\section{B Products of Binomials}

Lemma 8. Let $n$ and $t$ be positive integers. Let $x_{1}, x_{2}, \ldots, x_{t}$ be nonnegative integers with each $x_{i} \leq n$. Let $a$ and $b$ be the unique integers such that $\sum_{i} x_{i}=(t-b) a+b(a+1)$ and $0 \leq b \leq t-1$. Then

$$
\prod_{i=1}^{t}\left(\begin{array}{l}
n \\
x_{i}
\end{array}\right) \leq\left(\begin{array}{l}
n \\
a
\end{array}\right)^{t-b}\left(\begin{array}{c}
n \\
a+1
\end{array}\right)^{b}
$$

Proof. Choose $x_{1}, x_{2}, \ldots, x_{t}$ to maximise $\prod_{i}\left(\begin{array}{c}n \\ x_{i}\end{array}\right)$. Suppose on the contrary that two of the $x_{i}$ differ by at least two. Without loss of generality $x_{1} \geq x_{2}+2$. Let $x_{i}^{\prime}:=x_{i}$ except for $x_{1}^{\prime}:=x_{1}-1$ and $x_{2}^{\prime}:=x_{2}+1$. Thus $0 \leq x_{i}^{\prime} \leq n$. By assumption $\prod_{i}\left(\begin{array}{c}n \\ x_{i}\end{array}\right) \geq \prod_{i}\left(\begin{array}{c}n \\ x_{i}^{\prime}\end{array}\right)$. Hence $\left(\begin{array}{c}n \\ x_{1}\end{array}\right)\left(\begin{array}{c}n \\ x_{2}\end{array}\right) \geq\left(\begin{array}{c}n \\ x_{1}-1\end{array}\right)\left(\begin{array}{c}n \\ x_{2}+1\end{array}\right)$. It follows that $x_{1} \leq x_{2}+1$. This contradiction proves that all pairs of the $x_{i}$ differ by at most one. Thus $\prod_{i}\left(\begin{array}{c}n \\ x_{i}\end{array}\right)$ is maximised when $t-b$ of the $x_{i}$ equal $a$, and $b$ of the $x_{i}$ equal $a+1$. 